\title{
Simplifying Fitness Games for Users with Learning Disabilities
}

\author{
Liu Liu \\ Lancaster University \\ 1.liu@,lancaster.ac.uk
}

\author{
Patrick Stacey \\ Loughborough \\ University \\ p.stacey@1boro.ac.uk
}

\author{
Monideepa Tarafdar \\ Lancaster University \\ $\underline{\text { m.tarafdar@1ancaster.ac. }}$ \\ $\underline{\mathrm{uk}}$
}

\author{
Nikolaos Kourentzes \\ Lancaster University \\ n.kourentzes@1ancaster. \\ ac.uk
}

\begin{abstract}
Motivating people with learning disabilities (LD) to carry out physical exercises is a difficult task. Simplified fitness games can address this problem. Yet we do not know much about the design characteristics of the fitness games for this particular user group. Based on Rouse's process model, this paper explores the design characteristics in three development phases: 'conceptual outline', 'implementation' and 'outcome'. A mixed-method approach has been adopted. First, interviews and observations were conducted. Based on the qualitative findings and a literature review, a questionnaire was generated addressing the important design characteristics in each phases. The questionnaire surveyed 235 people from both game and healthcare industries to assess their agreement to the design characteristics. By identifying critical design characteristics in each phase, our paper provides guidance for an inclusive and nuanced approach to designing games for the users with LD. It identifies concepts in fitness games that intrinsically motivate physical activities.
\end{abstract}

\section{Introduction}

People with learning disabilities (LD) often lack physical exercise due to their impairments [1]. To change this situation, simplified fitness games can be helpful. Literature has shown that fitness games are effective in a healthcare context generally [2]. However, to the best of our knowledge, there is no research that studies fitness games within the more specific healthcare context of LD, let alone any putative design characteristics [3, 4]. Given the fact that people with LD in the UK often suffer from problems associated with obesity and physical activity [5], it is imperative to generate alternative tools, such as games, that can support and improve the quality of life for the LD users.

To study the design characteristics of fitness games in the LD domain, our study borrows Rouse's process model [6] and focuses on three game design phases 'conceptual outline', 'implementation' and 'outcome'. Especially, considering the user's condition and ability, this research explores the characteristics in the design phases that help developing simplified fitness games.

\section{Literature review}

In order to assess the design characteristics of fitness games in the LD domain, we first review the literature concerning the LD user group and how fitness games are applied in this context. This provides the conceptual foundation for our research.

\subsection{Challenges faced by people with learning disabilities}

A learning disability is defined as 'a significantly reduced ability to understand new or complex information or to learn new skills, a reduced ability to cope independently, and an impairment that started before adulthood, with a lasting effect on development' [7]. Although the UK is the only country that uses the term 'learning disabilities', other English speaking countries such as the USA and Australia use the term 'intellectual disabilities' [8]. In this paper, for consistency, we use the term 'learning disabilities'. There are four levels of LD: mild, moderate, severe and profound [8].

Mild LD refers to slight sensory or motor deficits [9]. Most of the people in this group are never diagnosed and are able to live independently [9]. They might need help with employment and housing or when under unusual stress [9]. People in the moderate LD group can talk and care for themselves under supervision [9]. Adults can undertake simple work [9]. People with severe LD have a slow pace of learning [9]. They may be able to communicate in a simple way [9]. They can perform easy tasks and engage in limited social interactions [9]. However, they often need help with daily activities and need to live under close supervision [8]. A person with profound LD usually has a number of disabilities 
which could include impairments to hearing, movement and vision. This can also include conditions such as epilepsy and autism [8]. People with severe LD would often need help with daily activities [8]. Their behaviors could be challenging for others [10]. They find it very difficult to communicate with others [10]. As a consequence, this group of people have been neglected and excluded from society and there is need to increase meaningful social interactions [11].

In general, people with LD exhibit poor fitness performance in terms of strength, endurance, and motor coordination [12]. Research has shown that this low performance is associated with limited motor development, sedentary lifestyle, mental impairments and short attention span [12]. Lack of motivation is also a cause for low levels of fitness [13]. Their physical performance is influenced by level of LD, for example, athletes with lower LD level perform better in motor coordination tests [14].

In terms of their mental conditions, people with LD struggle from mental health difficulties more than the general populations [15]. They often withdraw themselves from the environment, engage in obsessive or compulsive behaviors that would stop them from participating in everyday activities, and have low self-esteem [15].

Overall, people with LD struggle with physical movements, mental illness and low ability to learn. In this research, the opinions of all levels of LD for fitness games were surveyed. Considering that many people with LD have limited ability to read and write [9], the survey was carried out among the healthcare professionals who have sufficient knowledge of the needs of this particular group [16]. To distinguish different levels of LD, the healthcare professionals who participated in the survey were asked to choose the level they mostly deal with. All their answers to survey questions were given according to the chosen LD level.

\subsection{Fitness games for users with learning disabilities}

A fitness game is a video game that is used as a form to promote physical activities [17]. Examples of some successful commercial fitness games include: Wii Fit, Just Dance, Zumba Fitness, My Fitness Coach and Kinect Sports [2]. Research has shown that an increase of moderate intensity physical activity has a positive result in improving health [5]. Particularly for disabled populations, performing specially adapted exercises can change their current physical inactive situation [18].
Doing physical exercise not only helps people be stronger, but also contributes to decreasing anxiety and depression [19]. Additionally, regular physical activity promotes social inclusion and a sense of belonging [19]. Through body movements, people with LD can communicate their feelings to others [20] which they would struggle with verbally.

However, conventional fitness training programs are not always useful or appropriate for meeting the needs of people with LD [3]. In addition to their physical and psychological impairments, people with LD face a range of specific challenges including low motivation and little access to health care [3]. To promote physical exercise, fitness programs with motivational factors are recommended [21].

Fitness games have been tested to be effective in promoting physical exercise for adults with LD [3]. For school children with LD, fitness games have also been tested to be a success in physical education [4]. Combining exercise with computer games creates immersive and motivating training sessions [22]. When fitness games are designed for LD, they encourage players to repeat daily movements and help them improve in an enjoyable and virtual simulated environment [23]. Meanwhile, playing fitness games can help users build up self-esteem, confidence [24]. Play fitness games in groups also helps users connect [25] and change the isolated situation that the people with LD are facing.

Because of the special physical and mental conditions of this particular user group, fitness games have to be simplified. To discuss the design characteristics of simplified fitness games, this research focuses on the three typical phases based on Rouse's game design process model [6]: conceptual outline, implementation and outcome. There are many studies that process-map game design including the Boomerang [26], prototyping [27], as well as a variety of design techniques, like scenarios, body storming, paper prototyping, rapid prototyping, theatrical techniques of improvisation [28, 29], simulation [30], cuisinart [6], and play environments such as mixed reality [30]. Among all the process models, Rouse summarized a typical path that is easy to implement [6]. By adopting it in fitness game design, our research provides practical guidance for the industry. Additionally, Rouse's process model is user-orientated [6] which is critical when designing for LD users, given the sensitivity of their condition.

In the conceptual outline phase, game designers should focus on learning about user requirements and understand the associated game features. Designers have to decide the challenges in a game and the virtual environment to match these challenges. Besides, the pace of a game needs to be decided 
whether it is going to be slow or tense. Moreover, the rewards for players have to be considered. [6]

In the implementation phase, designers firstly need to build a game architecture to satisfy the aims and features proposed in the first phase. The next step is to design game mechanics and refine them until they are perceived as being fun. Designers also need to choose the right forms to display the game and use suitable technology to interact players with the virtual environment. With regards to human-computer interaction (HCI), the emphasis is on game interface design and visual adaptability [31]. When a game is finished, playtesting is required to collect feedback for further improvements. [6]

In the outcome phase, a game is expected to engage players by providing them with enjoyment, challenges, social interactions, emotional experiences and aesthetics [6]. This research focuses on the intrinsic outcomes that a fitness game brings because such outcomes are long-lasting when it comes to engaging players.

In summary, fitness games are able to help people with LD by motivating them to perform physical exercises and improving their mental status. Considering the special conditions of this user group, fitness games are required to be simplified to enable enjoyable gameplay and exercise. To design such fitness games, this research adopts a mixed-method to understand what their characteristics and features should be. The next section explains the research methods in this study.

\section{Methods}

This section discusses the mixed-method research design adopted for this study.

Combining qualitative and quantitative methods in this study is appropriate because of the complexity involved [32]. By combining both methods, researchers can: (i) confirm and corroborate each other's work via interplay, (ii) discover greater detail that develops the theories, (iii) generate new ways of thinking, and (iv) expand the depth and scope of the study [32]. There are many ways to combine these two methods and one of the combinations is demonstrated below [32]:

QUALITATIVE $\rightarrow$ QUANTITATIVE

(exploration) (confirm and deepen findings)

When applied to this study, we started with a qualitative case study based on interviews and observations for developing a fitness game named Somability. It provided insights into the perspectives of both game designers and product users. After analyzing experiences of those that developed
Somability, we learned that the three design phases suggested in the literature needed to be further clarified, explained and detailed, for the specific context of designing simplified fitness games for users with LD. To complement and extend the qualitative findings and confirm the results with a broader audience, the second phase of the study used a quantitative questionnaire-based method to understand the details of each design phase. The questionnaire was designed according to qualitative findings and literature. Survey respondents provided their perceptions of appropriate design characteristics in each phase for fitness game. Overall, a combination of methods enabled us to access richer data and provided a basis for a detailed and comprehensive analysis.

The next two sections go into more details about each study.

\section{Qualitative study}

The first phase of the research is a qualitative case study. The process involved working closely alongside a game company developing fitness games for users with LD. Ten interviews and three natural observations were conducted which helped discover three fundamental phases of designing simplified fitness games.

\subsection{Case description}

Somability is a fitness game that was produced by Cardiff Metropolitan University in partnership with Cariad Interactive. It contains three games: reach, balance and flow. Reach is a game that encourages users to reach high with their reflections to touch the shapes on the screen. The balance game requires users to open their arms and to balance as many digital balls as possible. Flow is a task-free game that allows users to perform any movements they like. There are three modes in Somability: mirror, stick man and shadow. The mirror mode shows users' original reflection on the screen. Under the stick man mode, users see their digital images as colorful skeletons. The shadow mode turns the digital images into colorful shadows and attaches beautiful lines to user's digital image. Users can choose any mode within the three games.

\subsection{Qualitative data collection}

In order to collect data for this paper, ten people involved with the development of this game were interviewed about their contribution to the development of Somability. The occupations of the 
interviewees were programmer, graphic designer, manager, researcher, dancer, facilitator and care giver. Each of them contributed to developing Somability in a different way. Interviewing them helped understand the design of the fitness game as a whole.

Additionally, three natural observations were taken in order to learn about the development team working. The first time involved travelling to the game studio and observing the game designers develop the game. The remaining observations focused on beta testing which was accomplished by designers with collaboration of users from a day care center. The last two observations involved not only game designers but also product users. The users' responses to the game helped analyzing the design process. In all we observed 26 people.

\subsection{Qualitative findings}

The analysis of the interview transcripts and the observation reports was conducted with the help of the qualitative data analysis software NVivo. Initial coding of the text was applied and 55 nodes were produced. These nodes were then linked and categorized according to the different phases of Somability's development process.

4.3.1. Design phase 1: conceptual outline. Interviews with the Somability team stated that during the conceptual outline phase, game designers conducted comprehensive user analysis to find out the essential functions of a fitness game. In order to do this, game designers as well as experts from LD communities were involved working together through role-playing, rehearsal and performance. This allowed the team to discover the idiosyncrasies of individual service user's needs, and thus find basic daily movements that could engage anyone even with limited movability. In order to avoid over complicated design and to make the software accessible for everyone, the simplicity principle was raised and was kept towards the end. The simplicity principle required designers to only use the basic game elements to minimize confusion and stress for users.

Somability also intended to improve user's mental states. During the conceptual outline design phase, experts from day care centers expressed the needs for users to socialize. Through observing potential users, game designers agreed that movements in fitness games should be designed to enable users to play together. Another user requirement raised by healthcare professionals was that games should enable users to decrease anxiety through exercise.
In summary, in order to simplify the fitness game, Somability focused on two functions of the game. On the physical side, the game's primary goal was to motivate exercise by repeating basic daily movements. On the psychology perspective, Somability aimed to bring users together by a group play game, and they also allowed users to decrease anxiety in free movements. From the case study, the first phase 'conceptual outline' is defined as the design period to decide fitness games' functions specific for the LD context.

4.3.2. Design phase 2: implementation. In the second design phase, implementation, Somability satisfied user requirements and designed a simplified fitness game. The three settings in Somability reach, balance and flow all have easy rules and clear instructions. All the responses in the games are positive and even when users fail in a game task, they would not receive negative feedback. Besides, Somability has no time limit which encourages users to repeat their movements to the extent that they are satisfied. By allowing mistakes and repetitive play, Somability promoted physical exercise.

Another design aspect that helped simplify the game was to make the interface clear. Somability offered users a clear interface by removing clutter and only providing the bare essential elements on the screen.

Overall, Somability tried to simplify the mechanics and interface in the implementation phase. Feedback from users and their care givers have shown that easy rules, repetitive play and a clear interface are effective in fitness games. The second phase 'implementation' is the design period to simplify fitness games through game mechanics.

4.3.3. Design phase 3: outcome. In the third design phase, outcome, Somability tried to intrinsically motivate users to perform physical exercise. In addition to being driven by the game concepts in Somability, users were also motivated to stay playing because of other achievements such as improvements of independent and social interaction. Users of Somability felt in control because the game offered instant feedback that tracked their progress. They became more interactive with others because Somability brought users together to play in groups. In addition to this, the game provided a competition aspect when encouraging users to build more flowers on the screen. Besides, care givers have also pointed out that because everyone wants to have a go in front of the machine, there is often healthy competition.

By matching the results of playing fitness games with users' intrinsic needs, this study explores means 
to design fitness games in order to intrinsically promote physical exercise. The third phase 'outcome' is defined as the design period that fitness games motivate users intrinsically.

In summary, conceptual outline, implementation and outcome were discovered to be the three design phases to make simplified fitness games for users with LD. To explore these concepts in the contextual details, the research uses questionnaires to gather further insights from a broader audience.

\section{Quantitative study}

Based on findings from both the qualitative data and literature research, a survey was created to target additional game designers and healthcare professionals. The purpose of the quantitative study was to extend the qualitative study and to learn about the detailed design characteristics in each development phase.

\subsection{Hypotheses}

In the conceptual outline phase, it is important to find out user requirements and thus design simplified game functions accordingly. For fitness games, the primary goal is to motivate exercise which is reflected in the case study analysis. Besides, interviewees expected fitness games to help users decrease depression. Fitness games can contribute to that because they are helpful in making users happier, healthier, and more open to others [3]. Additionally, fitness games involve users in various tasks and allow them to perform successfully, thus help users gradually build up self-esteem and confidence [33]. The first hypothesis is built around the design characteristics in the conceptual outline phase. To examine this hypothesis, four sub-hypotheses H1a H1d were generated (Table 1).

Hypothesis 1: in the conceptual outline phase, the functions of fitness games should be designed specific to the LD context.

To implement game functions, the fitness games should be based around simple concepts with clear instructions. Somability is designed with a high tolerance of mistakes and repetitive play; prior research shows that games designed for users with LD should allow them to process on their own rate and to repeat actions whenever they want [34]. Case study analysis also shows that the interface of fitness games should be specially designed to provide a clear and forgiving virtual environment. When designed appropriately, the game's interface can provide visual cues which offer clear and immediate feedback [35].
Technology in fitness games simplifies the games and supports users. It uses visual, auditory, and tactile cues to improve user experience [36]. By adding tactile and non-tactile features, fitness games can simplify the means to control games. For the purpose of this study, the game mechanics including game rules, instructions and interfaces are discussed. The second hypothesis is about the game mechanics in the implementation phase. There are five sub-hypotheses $\mathrm{H} 2 \mathrm{a}-\mathrm{H} 2 \mathrm{e}$ built to examine this hypothesis (Table 1).

Hypothesis 2: in the implementation phase, game mechanics should be used to simplify fitness games for people with LD.

An ideal fitness games drives users to exercise intrinsically out of interests and enjoyment. Providing intrinsic motivation is important because it changes user's behavior in the long term. This research borrows ideas from Self-Determination Theory (SDT) which suggests that the more control someone has over their decisions, the more likely they will be internally motivated to perform those actions. The three core facilitators in SDT are autonomy, relatedness and competence. Autonomy can be applied to fitness games by offering flexible game variation and utilizing positive feedback with the aid of clear instructions. Relatedness can be strengthened by making the connection between users more secure, frequent and robust. [37]

This research looks at how autonomy and relatedness aspects of fitness games can enrich the game itself. Additionally, interviews reflect that Somability created competition which motivated users to play more actively. In theory, all games are competitive because players compete with each other or against a game system [38]. While winners of a game receive a sense of achievement, losers can also enjoy the game play provided that they are given positive feedback [39]. Moreover, prior research has shown that for people with disabilities, games have other psychological benefits such as improving confidence, self-esteem and enjoyment [40]. With regards to the outcome phase, we developed hypothesis 3, to address the role of intrinsic motivations in fitness games which is examined through the four sub-hypotheses $\mathrm{H} 3 \mathrm{a}-\mathrm{H} 3 \mathrm{~d}$ (Table 1).

Hypothesis 3: in the outcome phase, fitness games for people with LD should be designed to intrinsically motivate users.

As shown in Table 1, all the sub-hypotheses were generated from qualitative findings and literature research results. Survey participants expressed their opinion about the importance of each sub-hypothesis with a five-point Likert scale: 1-strongly disagree, 2disagree, 3-neutral, 4-agree, 5-strongly agree. Some 
blank space was left on the questionnaire for participants to make additional comments.

Table 1. Phases, sub-hypotheses and sources

\begin{tabular}{|c|c|c|}
\hline Phases & Sub-hypotheses & Sources \\
\hline \multirow[t]{2}{*}{$\begin{array}{l}\text { Conc- } \\
\text { eputal } \\
\text { outline }\end{array}$} & $\begin{array}{l}\text { Fitness games should: } \\
\text { H1a: promote physical exercise } \\
\text { H1b: encourage social connections }\end{array}$ & $\begin{array}{l}\text { Qualita- } \\
\text { tive } \\
\text { findings }\end{array}$ \\
\hline & $\begin{array}{l}\text { H1c: develop users' self-esteem } \\
\text { H1d: decrease users' anxiety }\end{array}$ & $\begin{array}{l}{[3]} \\
{[33]}\end{array}$ \\
\hline \multirow[t]{2}{*}{$\begin{array}{l}\text { Impl- } \\
\text { emen- } \\
\text { ation }\end{array}$} & $\begin{array}{l}\text { Fitness games could be simplified } \\
\text { through: } \\
\text { H2a: allowing mistakes }\end{array}$ & $\begin{array}{l}\text { Qualita- } \\
\text { tive } \\
\text { findings }\end{array}$ \\
\hline & $\begin{array}{l}\text { H2b: allowing repetitive play } \\
\text { H2c: a clear interface } \\
\text { H2d: tactile features } \\
\text { H2e: non-tactile features }\end{array}$ & $\begin{array}{l}{[34]} \\
{[35]} \\
{[36]}\end{array}$ \\
\hline \multirow[t]{2}{*}{$\begin{array}{l}\text { Outc- } \\
\text { ome }\end{array}$} & $\begin{array}{l}\text { During game play, users should: } \\
\text { H3a: start to play with others } \\
\text { H3b: become more independent }\end{array}$ & $\begin{array}{l}\text { Qualita- } \\
\text { tive } \\
\text { findings }\end{array}$ \\
\hline & $\begin{array}{l}\text { H3c: compete with each other } \\
\text { H3d: feel happier }\end{array}$ & $\begin{array}{l}{[39]} \\
{[40]}\end{array}$ \\
\hline
\end{tabular}

\subsection{Quantitative data collection}

The market for fitness games targeted at learning disabilities is very new and therefore there are not many existing products. Thus only a handful of people have experience designing these types of games. To access a larger audience, the survey was carried out among both game designers and healthcare professionals.

Game designers were the obvious initial choice because of their familiarity with designing games and the experiences they could share when adapting to fitness games. Questionnaires were distributed during two game events where game designers from various game studios gathered. Participants included game writers, graphic designers, game producers and games studio managers.

Healthcare professionals provided emphasis on the topic of LD and their inputs were extremely useful for their knowledge of user requirements. Because many people with LD have trouble with writing and communication [16], they are not directly surveyed. Instead we surveyed healthcare professional who work with people with LD and thus intimately know their conditions and needs. Most participants were care givers who worked in care homes that specialized in LD. 30 care homes were visited to collect questionnaire responses. Occupations in this sector included nurses, care givers, social workers and care homes managers. In addition to this, healthcare professionals such as teachers, council workers, charity organization employees and researchers in this discipline were also involved.

The two groups of experts used the same questionnaire but their responses were separate so as to compare and contrast findings across groups. Altogether, there were 245 responses with a response rate of $41.8 \%(245 / 586) .114$ feedback were collected from game designers and 131 responses from healthcare professionals, generating response rates of $44.7 \%(114 / 255)$ and $39.6 \%(131 / 331)$ respectively. After screening, 10 surveys were removed due to missing data, leaving 235 samples.

\subsection{Quantitative findings}

To find out survey respondents' opinions about the three design phases, the response of each subhypothesis was compared with 3 (neutral). Considering that there were two participant groups, the similarity between the two groups was tested first with Mann-Whitney tests. This test was used because the answers of all questions were left-skewed instead of normally distributed. For the sub-hypotheses that received similar answers from the two groups ( $p$ value greater than .05), both groups' answers were combined. The median of the combined answer was compared with 3 using one-sample Wilcoxon Signed Rank Tests. If the p-value was smaller than .05 then there was enough evidence to support that the subhypothesis was significantly more positive than 3 . Regarding the sub-hypotheses that received different answers from the two groups (p-value smaller than .05), the median of each group was tested separately against 3 with one-sample Wilcoxon Signed Rank Tests.

Table 2. Sub-hypotheses that have similar values across two survey groups

\begin{tabular}{|l|l|l|l|}
\hline $\begin{array}{l}\text { Sub- } \\
\text { hypotheses }\end{array}$ & $\begin{array}{l}\text { Means of } \\
\text { healthcare } \\
\text { professionals }\end{array}$ & $\begin{array}{l}\text { Means of } \\
\text { game } \\
\text { designers }\end{array}$ & $\begin{array}{l}\text { Means of } \\
\text { combined } \\
\text { groups }\end{array}$ \\
\hline H1a & 4.26 & 4.06 & 4.17 \\
\hline H1c & 4.26 & 4.05 & 4.16 \\
\hline H1d & 4.11 & 4.15 & 4.13 \\
\hline H2b & 4.03 & 3.90 & 3.97 \\
\hline H2e & 3.75 & 3.60 & 3.68 \\
\hline H3a & 3.90 & 3.96 & 3.93 \\
\hline H3b & 4.28 & 4.27 & 4.28 \\
\hline
\end{tabular}

Table 3. Sub-hypotheses that have different values across two survey groups

\begin{tabular}{|l|l|l|l|}
\hline $\begin{array}{l}\text { Sub- } \\
\text { hypotheses }\end{array}$ & $\begin{array}{l}\text { Means of } \\
\text { healthcare } \\
\text { professionals }\end{array}$ & $\begin{array}{l}\text { Means of } \\
\text { game } \\
\text { designers }\end{array}$ & $\begin{array}{l}\text { Means of } \\
\text { combined } \\
\text { groups }\end{array}$ \\
\hline $\mathrm{H} 1 \mathrm{~b}$ & 4.38 & 3.50 & 3.97 \\
\hline $\mathrm{H} 2 \mathrm{a}$ & 3.89 & 4.35 & 4.11 \\
\hline $\mathrm{H} 2 \mathrm{c}$ & 4.20 & 4.65 & 4.41 \\
\hline $\mathrm{H} 2 \mathrm{~d}$ & 4.13 & 3.76 & 3.96 \\
\hline
\end{tabular}




\begin{tabular}{|l|l|l|l|}
\hline H3c & $3.30^{*}$ & $2.71^{*}$ & $3.02^{*}$ \\
\hline H3d & 4.48 & 4.73 & 4.59 \\
\hline
\end{tabular}

*Means for the 'competition' sub-hypotheses

Table 2 and Table 3 summarize the responses of all sub-hypotheses. The two survey groups gave similar marks for the seven sub-hypotheses in Table 2. All the marks were significantly higher than 3 which demonstrate that survey participants thought these design characteristics were of great importance when simplifying fitness games for LD users. For the other six sub-hypotheses in Table 3, the two groups of experts responded differently. But other than the 'competition' sub-hypothesis (H3c), all other subhypotheses provided feedback higher than a value of 3 which supported all of them. In summary, the key design characteristics that were significant according to the data collected and analyzed are following:

In the conceptual outline phase, in addition to the primary purpose, motivating physical exercise, fitness games should also be designed to promote social connections and decrease anxiety. During game play, users of fitness games should be able to increase their self-esteem.

With these design concepts in mind, designers will adopt simplified mechanics and assistive technology in the implementation phase. The mechanics in fitness games for LD users should allow mistakes and repetitive play. The technology in games should be able to assist users' special condition. In order to do that, tactile and non-tactile features in a clear interface will be helpful.

Simplified fitness games not only make users healthier but also improve their mental and social conditions; as a result, users are intrinsically motivated to continue playing. Experts have supported that happiness, independence and social skills will grow during game play. However, a common game element, competition, has to be handled carefully and to be kept in a safe level.

Considering that there are four levels of LD and those in each level group might have different opinion about fitness games, the survey data collected from healthcare professionals was split into four groups and further analyzed. This is feasible because participants from healthcare industry were asked to indicate the group of LD that they mostly deal with and then answer the questionnaire accordingly. After comparing data from four groups, there is no significant difference or trend. Therefore the conclusion made before is valid for all LD groups.

In conclusion, the design characteristics in the conceptual outline and implementation phases have been validated by testing the nine sub-hypotheses $(\mathrm{H} 1 \mathrm{a}-\mathrm{H} 1 \mathrm{~d}, \mathrm{H} 2 \mathrm{a}-\mathrm{H} 2 \mathrm{e})$. As for the design elements in the outcome phase, three sub-hypotheses (H3a, $\mathrm{H} 3 \mathrm{~b}, \mathrm{H} 3 \mathrm{~d}$ ) were tested to be correct. Autonomy and relatedness motivations were supported by both respondent groups, but game designers thought users should not be encouraged to compete (H3c).

\section{Discussion and contributions}

This section explores the differences and similarities across the two data sets in the quantitative study, as well as the links between qualitative and quantitative studies. Contributions of this research to theory and practice are also included.

\subsection{Comparison across the two respondent groups in the quantitative study}

With regards to the conceptual outline phase, survey response groups agreed on three subhypotheses (H1a, H1c, H1d) out of four. The only sub-hypothesis (H1b) that had split opinions was about whether fitness games should encourage social interactions. According to their comments on the questionnaire, game designers were concerned about the vulnerability of users with LD. Therefore they marked this sub-hypothesis (H1b) averagely 3.5, which is only slightly more than 3 . But healthcare professionals wanted fitness games to be a conduit for connecting users with others, especially given that they often withdraw themselves from others. Prior research has shown the effectiveness of games when it comes to increasing social interaction [3, 19, 25]. Fitness games with group play element are able to help change the isolated situation that this user group is suffering. However, users should interact with people they trust and always do it under supervision [9].

For the sub-hypotheses concerning the implementation phase, two (H2b, H2e) had similar feedback and the other three (H2a, H2c, H2d) did not. For the three sub-hypotheses that had different feedback from two response groups, two subhypotheses 'allowing mistakes' (H2a) and 'a clear interface' (H2c) had significantly higher marks from game designers in comparison to healthcare professionals. This indicates that when simplifying fitness games, healthcare professionals were most considerate about the limited abilities of the users; therefore they put great emphasis on forgiving game concepts [34] and a clear interface [35]. Given the equally high importance placed on this by the two respondent groups, both aspects were seen as compulsory. The other sub-hypothesis 'tactile features' (H2d) had a higher average score from 
healthcare professionals than game designers. Game designers thought it was not necessary and would add more cost. But given that all the answers in separate groups were significantly more than 3, results actually suggested an overall agreement on the subhypotheses ( $\mathrm{H} 2 \mathrm{a}, \mathrm{H} 2 \mathrm{c}, \mathrm{H} 2 \mathrm{~d})$.

Regarding the four sub-hypotheses concerning about the outcome phase, two survey response groups agreed on two sub-hypotheses (H3a, H3b) and disagreed on the other two (H3c, H3d). The subhypothesis 'feel happier' (H3d) had highest marks from both groups, even though there is a significant difference. Simplified fitness games should be able to receive this outcome because simple game concepts, forgiving game rules and clear interfaces will make users feel relaxed and enjoy the gameplay. On the contrary, the sub-hypothesis 'compete with each other' (H3c) had lowest marks from both response groups. Game designers had a mark (2.7) that was significantly lower than 3 , indicating that they thought competition should be totally avoided. Healthcare professionals thought competition was not a bad thing (3.3, significantly higher than 3) but needed to be handled with care; providing a positive outcome even for losers of the game. As a result, applying a competitive nature to fitness games appeared to be a delicate topic pointing to the idea that it could be applied but with great caution.

\subsection{Comparison between qualitative and quantitative findings}

After comparing the findings from qualitative and quantitative analysis, it became clear that most of the quantitative data supported the qualitative findings.

Perhaps the greatest difference between the qualitative and quantitative findings was the topic of competition. Somability encouraged users to compete with each other in a game to produce flowers on the screen by clapping hands; the user with the louder clapping would have more flowers. Somability demonstrated a successful attempt at applying competition to motivate users to partake in physical activity. However, survey respondents, in particular game designers, thought that competition among users with LD should be limited. One survey respondent commented on the questionnaire saying 'I like everyone to be a winner. So no one gets disappointed and resents using the game'. This worry is reasonable because research has shown that competition in games drives players to a more goaloriented behavior, which has been tested to have a negative effect on social and body engagement [41]. Besides, a player who is behind in an unbalanced competition might quit because a lead is overwhelming [23].

Although winners are always motivated to carry on playing, this does not necessarily mean that losers are discouraged completely. The losers of games can be intrinsically motivated if they were offered positive feedback [39]. With that said, fitness games could apply the idea of providing positive responses such as the sounds of applause when users make progress. Besides, users should be encouraged to compete against themselves but not against others. In addition, designers can motivate users to play by other methods instead of competition, for example, the enjoyable experience of exploring the virtual game environment [22].

Overall, the research suggests that it is best for fitness games to avoid unhealthy competition. However, adding positive feedback in fitness games can help motivate both winners and losers. Users with LD should be guided to compete with themselves.

\subsection{Contributions to theory and practice}

This research contributes to literature by making a theoretical connection between fitness game design and inclusive design. It identifies and describes three key design phases for simplified fitness games, critical for users with LD. There is evidence to suggest that the design characteristics associated with these phases have been verified. The paper also emphasizes the intrinsic motivations in fitness games. It does so by combining Self-Determination Theory with game design theories to generate ways in which intrinsic motivations can be assessed during gameplay. Among the intrinsic motivations, we highlight the important and sensitive topic of the use of competition in fitness games and points out that it should be handled with caution. Further, this study emphasizes the involvement of expert practitioners such as game designers and healthcare professionals in evaluating the usefulness of design guidelines in this delicate context. In contrast to prioritizing engagement of end users in evaluating HCI design [42], in this particular context of designing for LD, practitioners such as healthcare professionals are equally important and provide critical perspectives on the design element.

In a practical sense, this research outlines the key design concepts of a successful fitness game and it potentially contributes to the quality of life of people with LD. Research on fitness games brings to this user group, physical, mental and social benefits as well as entertainment opportunities [42]. For game designers, the design characteristics proposed can 
help them avoid previously identified pitfalls. Such guidelines provide designers with a structured approach to make fitness games for LD users. Moreover, this research breaks down the boundary between researchers, commercial game designers and healthcare professionals. Researchers are encouraged to collaborate with expert practitioners when developing design guidelines so as to make conceptual theories more applicable to targeted field. In return, practitioners are recommended to involve in the development of design guidelines to help advancing knowledge.

\section{Conclusion}

This paper used a mixed method approach to investigate the three design phases of 'conceptual outline', 'implementation' and 'outcome' with a focus on simplified fitness games for LD users. Findings from both the qualitative study and the quantitative study support the idea that such games should consider user's physical, mental, social and motivational needs. Our paper therefore proposes and provides guidance for an inclusive and nuanced approach to designing games for people with LD that is sensitized to their specific conditions and requirements.

\section{References}

[1] P. R. Messent, C. B. Cooke, and J. Long,. "Daily physical activity in adults with mild and moderate learning disabilities: Is there enough?", Disability and Rehabilitation, 20(11), 1998, pp. 424-427.

[2] S. McCallum, "Gamification and serious games for personalized health." Stud Health Technol Inform 177, 2012, pp. 85-96.

[3] M. S. Lotan, Yalon-Chamovitz, and P. L. T. Weiss. "Improving physical fitness of individuals with intellectual and developmental disability through a Virtual Reality Intervention Program." Research in developmental disabilities 30.2, 2009, pp. 229-239

[4] S. X. Cai, and A. S. Kornspan. "The use of exergaming with developmentally disabled students." Strategies 25.3, 2012, pp. 15-18.

[5] J. Robertson, E. Emerson, N. Gregory, C. Hatton, S. Turner, S. Kessissoglou, A. Hallam., "Lifestyle related risk factors for poor health in residential settings for people with intellectual disabilities." Research in developmental disabilities 21.6, 2000, pp. 469-486.

[6] R. Rouse III, Game design: Theory and practice. Jones \& Bartlett Learning, 2010.
[7] G. Martin, "'Valuing people'--a new strategy for learning disability for the 21 st century: how may it impinge on primary care?." Br J Gen Pract 51.471, 2001, pp. 788790 .

[8] E. Hardie, and L. Tilly. An introduction to supporting people with a learning disability. Sage, 2012.

[9] N. Bouras, B. Murray, and T. Joyce, eds. Mental Health in Learning Disabilities: A Training Pack for Staff Working with People who Have a Dual Diagnosis of Mental Health Needs and Learning Disabilities. Associated Issues. Pavilion, 1995.

[10] J. Ware, "Ascertaining the views of people with profound and multiple learning disabilities." British Journal of Learning Disabilities 32.4, 2004, pp. 175-179.

[11] Sheehy, Kieron, and Melanie Nind. "Emotional well-being for all: mental health and people with profound and multiple learning disabilities." British Journal of Learning Disabilities 33.1, 2005, pp. 34-38.

[12] Š.Golubović, J. Maksimović, B. Golubović, and N. Glumbić, "Effects of exercise on physical fitness in children with intellectual disability." Research in developmental disabilities 33.2, 2012, pp. 608-614.

[13] J. W. Halle, D. Gabler-Halle, and Y. B. Chung. "Effects of a peer-mediated aerobic conditioning program on fitness levels of youth with mental retardation: two systematic replications." Mental retardation 37.6, 1999, pp. 435-448.

[14] L. Guidetti, E. Franciosi, M.C. Gallotta, G.P. Emerenziani, and C. Baldari, "Could sport specialization influence fitness and health of adults with mental retardation?." Research in developmental disabilities 31.5, 2010, pp. 1070-1075.

[15] P. Lacey, and C. Oyvry. People with Profound \& Multiple Learning Disabilities: A Collaborative Approach to Meeting. Routledge, 2013.

[16] J. D. Lin, J. L. Wu, and P. N. Lee. "Healthcare needs of people with intellectual disability in institutions in Taiwan: outpatient care utilization and implications." Journal of Intellectual Disability Research 47.3, 2003, p. 169-180.

[17] J. Sinclair, P. Hingston, and M. Masek.

"Considerations for the design of exergames." Proceedings of the 5th international conference on Computer graphics and interactive techniques in Australia and Southeast Asia. ACM, 2007.

[18] R.R. Pate, M. Pratt, S.N. Blair, W.L. Haskell, C.A. Macera, C. Bouchard, D. Buchner, W. Ettinger, G.W. Heath, A.C. King, and A. Kriska, "Physical activity and public health: a recommendation from the Centers for 
Disease Control and Prevention and the American College of Sports Medicine." Jama 273.5, 1995, pp. 402-407.

[19] V. A. Temple, and J. W. Walkley. "Perspectives of constraining and enabling factors for health-promoting physical activity by adults with intellectual disability." Journal of Intellectual and Developmental Disability 32.1, 2007, pp. 28-38.

[20] S. J. Biddle, K. Fox, and S. Boutcher, eds. Physical activity and psychological well-being. Routledge, 2003.

[21] Rogers-Wallgren, J. L., R. French, and V. Ben-Ezra. "Use of reinforcement to increase independence in physical fitness performance of profoundly mentally retarded youth." Perceptual and motor skills 75.3, 1992, p. 975-982.

[22] S. Mokka, A. Väätänen, J. Heinilä, and P. Välkkynen, "Fitness computer game with a bodily user interface." Proceedings of the second international conference on Entertainment computing. Carnegie Mellon University, 2003.

[23] T. Campbell, B. Ngo, and J. Fogarty. "Game design principles in everyday fitness applications." Proceedings of the 2008 ACM conference on Computer supported cooperative work. ACM, 2008.

[24] A. Grimes, V. Kantroo, and R. E. Grinter. "Let's play!: mobile health games for adults." Proceedings of the 12th ACM international conference on Ubiquitous computing. ACM, 2010.

[25] J. Yim, and T. C. Graham. "Using games to increase exercise motivation." Proceedings of the 2007 conference on Future Play. ACM, 2007.

[26] P. Stacey, and J. Nandhakumar. "Opening up to agile games development." Communications of the ACM 51.12, 2008, pp. 143-146.

[27] Y. Baba, , and F. T. Tschang. "Product development in Japanese TV game software: The case of an innovative game." International Journal of Innovation Management 5.04, 2001, pp. 487-515.

[28] C. Clanton, "An interpreted demonstration of computer game design." CHI 98 Cconference Summary on Human Factors in Computing Systems. ACM, 1998.

[29] D. Johnson, and J. Wiles. "Effective affective user interface design in games." Ergonomics 46.13-14, 2003, pp. 1332-1345.

[30] K. Kanev, and T. Sugiyama. "Design and simulation of interactive 3D computer games." Computers \& Graphics 22.2, 1998, pp. 281-300.

[31] S. J. Czaja, and C. C. Lee. Information technology and older adults. CRC Press, 2009.
[32] M. B. Miles, and A. M. Huberman. Qualitative data analysis: An expanded sourcebook. Sage, 1994.

[33] Y. Hutzler, and O. Korsensky. "Motivational correlates of physical activity in persons with an intellectual disability: a systematic literature review." Journal of Intellectual Disability Research 54.9, 2010, pp. 767-786.

[34] C. D. Coles, D.C. Strickland, L. Padgett, and L. Bellmoff, "Games that "work": Using computer games to teach alcohol-affected children about fire and street safety." Research in developmental disabilities 28.5, 2007, pp. 518530.

[35] K. Werbach, and D. Hunter. For the win: How game thinking can revolutionize your business. Wharton Digital Press, 2012.

[36] C. Nicolle, and J. Abascal. Inclusive design guidelines for HCI. CRC Press, 2001.

[37] R. M. Ryan, and E. L. Deci. "Self-determination theory and the facilitation of intrinsic motivation, social development, and well-being." American psychologist 55.1, 2000, pp. 68.

[38] K. Salen, and E. Zimmerman. Rules of play: Game design fundamentals. MIT press, 2004.

[39] M. Vansteenkiste, and E. L. Deci. "Competitively contingent rewards and intrinsic motivation: Can losers remain motivated?." Motivation and emotion 27.4, 2003, pp. 273-299.

[40] S. Yalon-Chamovitz, and P. L. T. Weiss. "Virtual reality as a leisure activity for young adults with physical and intellectual disabilities." Research in Developmental Disabilities 29.3, 2008, pp. 273-287.

[41] N. Bianchi-Berthouze, W. W. Kim, and D. Patel. "Does body movement engage you more in digital game play? And Why?." Affective computing and intelligent interaction. Springer Berlin Heidelberg, 2007. pp.102-113.

[42] K. Isbister, U. Schwekendiek, and J. Frye. "Wriggle: an exploration of emotional and social effects of movement." CHI'11 Extended Abstracts on Human Factors in Computing Systems. ACM, 2011. 